\title{
Las finalidades del conocimiento escolar en ciencias en las orientaciones curriculares del Ministerio de Educación Nacional de Colombia
}

Autor de correspondencia: ${ }^{1}$ amcardenas2@hotmail.com Recibido: 02 de diciembre de 2020 Revisado: 05 febrero de 20201 Aprobado: 30 de septiembre de 2021 Publicado: 23 de octubre de 2021

\author{
Ana María Cárdenas Navas ${ }^{1}$, Carmen Alicia Martínez Rivera \\ Universidad Distrital Francisco José de Caldas, Colombia
}

\section{Resumen}

Este artículo busca dar cuenta de las finalidades de las orientaciones declaradas en ciencias naturales expedidas por el Ministerio de Educación Nacional de Colombia. Esta es una de las categorías para la caracterización del conocimiento escolar de dichas orientaciones. El estudio se adelanta en el marco del Doctorado Interinstitucional en Educación. La investigación se desarrolló según el enfoque cualitativo desde una perspectiva interpretativa a través del método del estudio de caso. Se examinaron los Lineamientos curriculares, los Estándares básicos de competencias y los Derechos básicos del aprendizaje para el área de ciencias naturales y educación ambiental a través del análisis de contenidos con apoyo del software Atlas.ti. Como resultados se destacan: el aporte del conocimiento escolar en ciencias para la construcción de una ciudadanía responsable; el conocimiento científico como el principal referente de las finalidades; el propósito de desarrollar actitudes, prácticas y valores; y cómo el conocimiento científico se puede convertir en la base para continuar con la formación académica o para afrontar la vida laboral y productiva. Se plantean nuevas necesidades de investigación, por ejemplo, cómo relacionar estas finalidades de formación de ciudadanos con los contenidos propuestos.

Palabras clave: ciencias naturales, conocimiento escolar, educación en ciencias, orientaciones curriculares 


\title{
The purposes of school knowledge in science in the Colombian Ministry of National Education curricular guidelines
}

\begin{abstract}
This article aims to report on the purposes of the stated guidelines for natural sciences established by the Colombian Ministry of National Education. This is one of the categories for characterizing school knowledge in such guidelines. The project is carried out in the context of the Interinstitutional Doctorate in Education. The research was conducted according to the qualitative approach from an interpretative perspective through the case study method. The Curricular Guidelines, the Basic Standards of Competencies, and the Basic Learning Rights for the area of natural sciences and environmental education were examined through content analysis using the Atlas.ti software. The following results stand out: the contribution of school knowledge in science for the construction of a responsible citizenship; scientific knowledge as the main referent of the purposes; the aim of developing attitudes, practices, and values; and how scientific knowledge may become the basis to continue with academic training or to face working and productive life. Finally, new research needs arise, for example, how to relate these citizen training goals to the proposed contents.
\end{abstract}

Keywords: natural sciences, school knowledge, science education, curricular guidelines

\section{As finalidades do conhecimento escolar em ciências nas orientações curriculares do Ministério da Educação Nacional da Colômbia}

\section{Resumo}

Este artigo visa apresentar um relatório sobre as finalidades das diretrizes estabelecidas pelo Ministério da Educação Nacional da Colômbia para as ciências naturais. Esta é uma das categorias para a caracterização do conhecimento escolar em tais diretrizes. O projeto desenvolve-se no marco do Doutorado Interinstitucional em Educação. A pesquisa foi desenvolvida segundo a abordagem qualitativa a partir de uma perspectiva interpretativa através do método do estudo de caso. As Diretrizes Curriculares, os Padrões Básicos de Competências e os Direitos Básicos de Aprendizagem para a área de ciências naturais e educação ambiental foram examinados através da análise de conteúdo usando o software Atlas.ti. Destacam-se os seguintes resultados: a contribuição do conhecimento escolar em ciência para a construção de uma cidadania responsável; o conhecimento científico como principal referência das finalidades; o propósito de desenvolver atitudes, práticas e valores; e como o conhecimento científico pode se tornar a base para continuar com a formação acadêmica ou para enfrentar a vida profissional e produtiva. Finalmente, surgem novas necessidades de pesquisa, por exemplo, como relacionar estes objetivos de formação de cidadãos com os conteúdos propostos.

Palavras-chave: ciências naturais, conhecimento escolar, educação científica, diretrizes curriculares 
${ }^{1}$ El presente artículo da cuenta de los resultados de la categoría finalidades en el marco de la tesis doctoral que indaga por el conocimiento escolar en ciencias, declarado en los Lineamientos Curriculares. Área de Ciencias Naturales y Educación Ambiental (LCNEA) (Ministerio de Educación Nacional [MEN], 1998), los Estándares Básicos de Competencias. Ciencias Naturales (ECN) (MEN, 2006) y los Derechos Básicos del Aprendizaje. Ciencias Naturales (DCN) (MEN, 2016). En Colombia, a partir de la Ley 115 (1994), el MEN ha expedido orientaciones curriculares que tienen como propósito apoyar los procesos de construcción de PEI o de las áreas obligatorias y fundamentales en el marco de la autonomía institucional. En la tabla 1, para cada orientación, se ilustra la normativa que soporta su expedición y el plan de gobierno o plan sectorial del periodo en el que se elaboró:

\section{Tabla 1}

Orientación curricular, normativa o propuesta que la respalda y plan de gobierno o plan sectorial del periodo en el que se construye

\begin{tabular}{lll}
\hline \multicolumn{1}{c}{$\begin{array}{c}\text { Orientación } \\
\text { curricular }\end{array}$} & Normativa o propuesta & \multicolumn{1}{c}{ Plan de gobierno o plan sectorial } \\
\hline $\begin{array}{l}\text { LCNEA (MEN, } \\
1998)\end{array}$ & $\begin{array}{l}\text { Ley General de Educación } \\
(\text { Ley 115, 1994, art. 148) }\end{array}$ & $\begin{array}{l}\text { Plan Nacional de Desarrollo e Inversiones 1995- } \\
\text { 1998 (Ley 188, 1995), denominado Salto educativo. }\end{array}$ \\
\hline ECN (MEN, 2006) & Ley 715 (2001, art. 5) & $\begin{array}{l}\text { La revolución educativa. Plan sectorial 2002-2006 } \\
\text { (MEN, 2003). }\end{array}$ \\
\hline DCN (MEN, 2016) & Propuesta Siempre Día & $\begin{array}{l}\text { Plan Nacional de Desarrollo 2014-2018 } \\
\text { (Departamento Nacional de Planeación, 2015). Para } \\
\text { el sector educativo se propone una proyección hasta } \\
\text { el año 2025 con el eslogan "Colombia, la mejor } \\
\text { educada". }\end{array}$ \\
\hline
\end{tabular}

En la investigación en didáctica de las ciencias es relevante analizar desde los currículos oficiales de ciencias las finalidades. Esto se abordó desde diferentes líneas de investigación por autores como Goodson (1991) en Inglaterra, Merchán (2002) en España y Lopes (2005) en Brasil. En el contexto nacional, en los saberes escolares, se destacan los trabajos de Álvarez $(2007,2015)$ y los de Roa (2015) para el caso de la biología escolar.

El estudio de los documentos que soportan los currículos oficiales para la enseñanza de las ciencias - resoluciones, directrices, decretos, orientaciones, estándares, entre otros- ha sido un problema relevante de investigación, según lo manifiestan Lopes (2005), Criado et al. (2014), Martínez (2016), Cárdenas y Martínez (2018), entre otros.

Respecto al tema de análisis del conocimiento escolar en ciencias sobre documentos oficiales del currículo, se destaca la investigación de Lopes (2008), donde se reconocen particularidades del conocimiento escolar y se analiza la reforma de la educación secundaria, en el caso de la enseñanza de las ciencias, desde los documentos oficiales en Brasil. Allí se indica la articulación entre las asignaturas y las competencias como una construcción antagónica entre el currículo nuevo y el currículo antiguo, pues el antiguo es de carácter

\footnotetext{
1 Artículo de resultados. Proyecto de tesis doctoral El conocimiento escolar en los Lineamientos Curriculares, Estándares Básicos de Competencias y Derechos Básicos del Aprendizaje para el Área de Ciencias Naturales en Colombia: estudio de caso, en el marco del Doctorado Interinstitucional en Educación, Centro de Investigaciones y Desarrollo Científico — código CIDCUD 2460173720—, Universidad Distrital Francisco José de Caldas.

2 El MEN, mediante el Decreto 0325 del 25 de febrero de 2015, definió que los establecimientos de la educación básica y media, tanto de carácter público y privado, incorporarán en su calendario académico un día al año de receso estudiantil con el propósito de llevar a cabo la jornada de excelencia académica "Día E”. El propósito es que directivos docentes, docentes y personal administrativo revisen los resultados institucionales y definan un plan de mejora para alcanzar las metas. Esta propuesta cuenta con una caja de herramientas, dentro de la cual se ubican los DBA.
} 
"enciclopédico" y "memorista" y no posibilita enfrentarse a los desafíos actuales; mientras que el currículo nuevo, con un enfoque por competencias y el desarrollo de pensamiento crítico, permite la resolución de problemas contextualizados y se convierte en la base para los requerimientos de la sociedad y del trabajo.

También son relevantes las investigaciones realizadas por el equipo de Criado et al. (2014), donde analizaron los documentos que soportan currículos oficiales de la educación y alfabetización científica en el marco de la didáctica de las ciencias desde el ámbito estatal -España-, desde la comparación de la propuesta estatal con las de otros países - Inglaterra (Gales) y Estados Unidos-. Cabe destacar que, según Martínez et al. (2020), las investigaciones en el ámbito latinoamericano son escasas y, en cambio, hay una preeminencia de estos estudios en Estados Unidos. En Colombia, se retomaron, por un lado, la investigación que analiza el conocimiento escolar de tres orientaciones curriculares de la Secretaría de Educación de Bogotá (Martínez et al., 2016) y, por otro, el avance de una tesis doctoral, donde se caracterizó el conocimiento escolar del apartado "Estructura de los estándares básicos de competencias” del documento ECN (Cárdenas \& Martínez, 2017).

En este contexto, como lo indica García (1998), en el marco del conocimiento escolar es necesario: desde la perspectiva ideológica crítica, explicitar las intencionalidades que subyacen en la determinación del conocimiento escolar; desde la visión compleja, dar cuenta de ese sistema de ideas - el qué enseñar-que ubica, además de tipos de contenidos, su selección, organización, complejidad y secuenciación; y desde la perspectiva crítica, el reconocimiento de la diversidad de conocimientos válidos (Merchán \& García, 1994). En el contexto nacional, se ratifica lo anterior, a partir de la visión de Martínez (2017), cuando, desde las investigaciones del conocimiento profesional del docente de ciencias sobre el conocimiento escolar (Martínez, 2000, 2005, 2016; Martínez \& Valbuena, 2013), se avanza en la comprensión de las finalidades, los contenidos escolares, las fuentes y criterios de selección de estos contenidos, los referentes epistemológicos del conocimiento escolar y sus criterios de validación. Este artículo está centrado en el para qué del conocimiento escolar en ciencias de las orientaciones curriculares mencionadas.

Las finalidades del conocimiento escolar en ciencias son asunto de reflexión importante. Se analizó, por ejemplo, qué objetivos se plantean para el currículo de ciencias; cuáles son los principales cambios del para qué educar en ciencias; qué factores han influenciado estos cambios; cuál debería ser el aporte de la formación en ciencias en la sociedad actual; qué retos deberán asumirse desde el currículo; las prácticas de los maestros y los procesos de aprendizaje para trabajar en pro de esas finalidades; entre otros aspectos.

Frente a cuáles han sido los objetivos del currículo de ciencias, Bybee y Deboer (1994) se centran en tres: adquirir conocimiento científico; aprender procedimientos y metodologías de la ciencia; y comprender las aplicaciones del conocimiento científico en relación con la ciencia y la sociedad. Así, los retos contemporáneos plantean la viabilidad de propuestas globales y sistémicas que atiendan a las necesidades de formación científica de los estudiantes (Ottander \& Simmon, 2021; Roberts \& Bybee, 2014), perspectiva en la que el conocimiento del contexto y de la cultura son centrales (Martínez \& Molina, 2011). Paixao y Cachapuz (1999) proponen como importante formar ciudadanos científicamente alfabetizados, hecho que implica abordar de manera diferente la enseñanza de las ciencias. 
Desde otra mirada, Hodson (2003) analiza los eslóganes y cómo la alfabetización científica no logra satisfacer las necesidades, intereses o aspiraciones individuales ni de la sociedad, frente a lo cual propone dos ideas principales: una relacionada con el aporte que deberá hacer la alfabetización científica para formar en la acción sociopolítica; y otra para la toma de decisiones frente a los problemas sociales y ambientales. Furió et al. (2001), por su parte, en el estudio de las finalidades de la enseñanza de las ciencias para la secundaria obligatoria, al retomar a Hodson (1993), plantean que la alfabetización científica antes de la década de los 80 estuvo centrada en familiarizar a los estudiantes con las teorías, conceptos y procesos del conocimiento científico. Sin embargo, en las décadas de los 80 y de los 90 se amplía y se propone orientar la enseñanza de las ciencias hacia aspectos más personales y sociales.

Como lo plantean Gil y Vilches (2001, 2004), el conocimiento científico, la aproximación a la naturaleza de las ciencias, las prácticas científicas y el establecimiento de las relaciones entre ciencia, tecnología y sociedad (CTS) tendrán que estar al servicio de una toma fundamentada de decisiones, idea soportada a partir de lo propuesto por Aikenhead (1985). Como se aprecia, la educación científica pone el acento en aportar a la formación de ciudadanos y ciudadanas.

Respecto al énfasis en la ciencia escolar, Acevedo (2004) y Vázquez et al. (2005) indican, por una parte, la relevancia de la ciencia escolar en aspectos relacionados no solo con la vida diaria, sino también con el ejercicio de la ciudadanía y con la continuidad de estudios referentes a la ciencia y la vida laboral; y por otra, la importancia de la ciencia escolar con una orientación más humana, cuya base sean las relaciones CTS. Asimismo, Chamizo y Pérez (2017) indican la importancia de aclarar qué se entiende por alfabetización científica.

Respecto a aspectos de contribución de la educación científica en lo individual y en lo social, Lemke (2006) plantea que, desde el marco de la didáctica de las ciencias, se requiere:

- articular el compromiso emocional e intelectual frente a los fenómenos naturales.

- promover el pensamiento crítico acerca de los desarrollos de la ciencia y la tecnología.

- el diálogo del conocimiento científico con otras formas de conocimiento humano.

- analizar las implicaciones individuales y sociales de la creación, difusión, interpretación y utilización de diferentes tipos de textos.

Banet (2007), por su parte, explicita que el conocimiento científico debe hacer parte de la cultura de los ciudadanos a partir de un pensamiento crítico que se refleje en la vida diaria, en las decisiones de los desarrollos científicos y tecnológicos y en la forma de asumir, por ejemplo, las situaciones frente a la salud y el ambiente.

Hodson (2013), al retomar a Kyle (1996), plantea que el aporte de la educación en ciencias debe estar centrado en que los estudiantes aprendan a participar en acciones sociopolíticas, además de comprender las interacciones sociales, políticas y económicas de la actividad científica y tecnológica para poder asumir posiciones frente a problemas contemporáneos. En esta misma línea, Criado et al. (2014), desde un análisis comparativo de los currículos de ciencias de España, Gales y Estados Unidos, identifica que comparten unos mínimos para el caso de las finalidades de alfabetización científica: el desarrollo personal, la comprensión 
del mundo, el desenvolvimiento en la vida diaria y el desarrollo de pensamiento crítico frente a cuestiones sociocientíficas.

Como se puede apreciar, el conocimiento escolar en ciencias desde la reflexión del para qué ha enfatizado el rol del conocimiento escolar en la formación de ciudadanos y ciudadanas, reflejada principalmente en el desarrollo del pensamiento crítico con capacidad para tomar decisiones en pro del bienestar individual y social, fundamentadas en la participación activa frente a situaciones y problemáticas del entorno y en la comprensión más holística de lo que son e implican los desarrollos de la ciencia y de la tecnología en el entramado de relaciones sociales, políticas, económicas y culturales. Igualmente, hay un interés por el lugar del conocimiento científico en el contexto escolar, que deberá trascender su adquisición, el desarrollo de habilidades y actitudes científicas y la aproximación a la naturaleza de las ciencias hacia la articulación del conocimiento científico con otros conocimientos igualmente válidos que contribuyan a una comprensión mucho más holística de situaciones y fenómenos. En términos de Porlán (2018), la educación científica desde un paradigma emergente busca contribuir al desarrollo personal, con compromiso social y ambiental para favorecer una democracia epistemológica.

\section{Referentes metodológicos}

La investigación se soporta en la didáctica de las ciencias desde un enfoque cualitativo. Vasilachis (2006), al retomar a Mason (1996), señala para esta perspectiva las siguientes características:

a) [está] fundada en una posición filosófica ampliamente interpretativa en el sentido que se interesa en las formas en las que el mundo social es interpretado, comprendido, experimentado y producido, b) basada en métodos de generación de datos flexibles y sensibles al contexto social en el que se producen, y c) sostenida por métodos de análisis y explicación que abarcan la comprensión de la complejidad, el detalle y el contexto. (p. 25)

Igualmente, desde la perspectiva interpretativa, según los planteamientos de Vasilachis (2006) del paradigma interpretativo, el investigador "privilegia lo profundo sobre lo superficial, lo intenso sobre lo extenso, lo particular sobre las generalidades, la captación del significado y del sentido interno subjetivo, antes que la observación exterior de presuntas regularidades objetivas" (p. 49).

Teniendo en cuenta lo planteado por Stake (1999), la caracterización de las orientaciones curriculares de ciencias naturales expedidas por el MEN es un estudio de caso único de carácter intrínseco, pues el caso estaría conformado por los documentos - LCNEA, ECN y DCN- preseleccionados inicialmente por su carácter de orientación curricular desde el MEN para el área de ciencias naturales. El interés intrínseco está en poder dar cuenta del conocimiento escolar, tanto desde lo particular como desde la relación con las orientaciones curriculares en mención, para aportar a una de las necesidades identificadas en ese campo del conocimiento escolar.

La codificación se llevó a cabo con apoyo del software Atlas.ti, por sus bondades relacionadas con la construcción de unidades o segmentos asociados a palabras claves o códigos que se pueden mostrar de diferentes formas (Chernobilsky, 2006). Con base en el análisis textual discursivo, según los planteamientos de Moraes y Galiazzi (2006) y las 
recomendaciones de trabajos anteriores (Martínez, 2000, 2016; Martínez \& Valbuena, 2013), desde el análisis de contenido (Bardín, 1996), se desarrolló un proceso de codificación, elaboración de unidades de información, categorización, inferencias y establecimiento de relaciones.

Las subcategorías referentes a la categoría finalidades emergen del proceso de codificación, principalmente las relacionadas con las finalidades generales - que se identifican a partir de los fines de educación, de los objetivos por niveles y del papel de la escuela en Colombia-; mientras que las que dan cuenta del para qué del conocimiento escolar en ciencias - los apartados de cada orientación en donde se explicitan los objetivos, propósitos o metas de la educación en ciencias- se han enriquecido a partir de (1) de las investigaciones del conocimiento profesional de los profesores de ciencias sobre el conocimiento escolar (Martínez, 2000, 2016; Martínez \& Valbuena, 2013), (2) del conocimiento escolar y orientaciones curriculares para el área de ciencias naturales de la Secretaría de Educación de Bogotá (Cárdenas \& Martínez, 2018; Martínez et al., 2016), así como (3) de los trabajos del grupo de Criado et al. (2014) desde el campo de la ciencia escolar, donde se analiza el currículo oficial de ciencias. Es importante mencionar que el proceso de codificación fue triangulado por una de las investigadoras a partir de citas asignadas como "dudosas".

La propuesta de niveles de complejidad como un constructo teórico y metodológico, denominado hipótesis de progresión o hipótesis de progresión-transición, para el caso de los resultados, tiene su base en trabajos de Martínez (2000) a partir de su tesis doctoral y en otros trabajos con investigadores en el contexto colombiano. Como lo plantean Martínez y Valbuena (2013), esta propuesta supone la existencia de un nivel inicial — visión simplehasta un nivel de referencia flexible - visión compleja-, con ciclos y fluctuaciones propios del proceso.

La tabla 2 muestra subcategorías de la categoría finalidades generales para la educación básica y media y su respectiva complejidad.

\section{Tabla 2}

Niveles de complejidad. Subcategorías referidas a finalidades generales para la educación básica y media

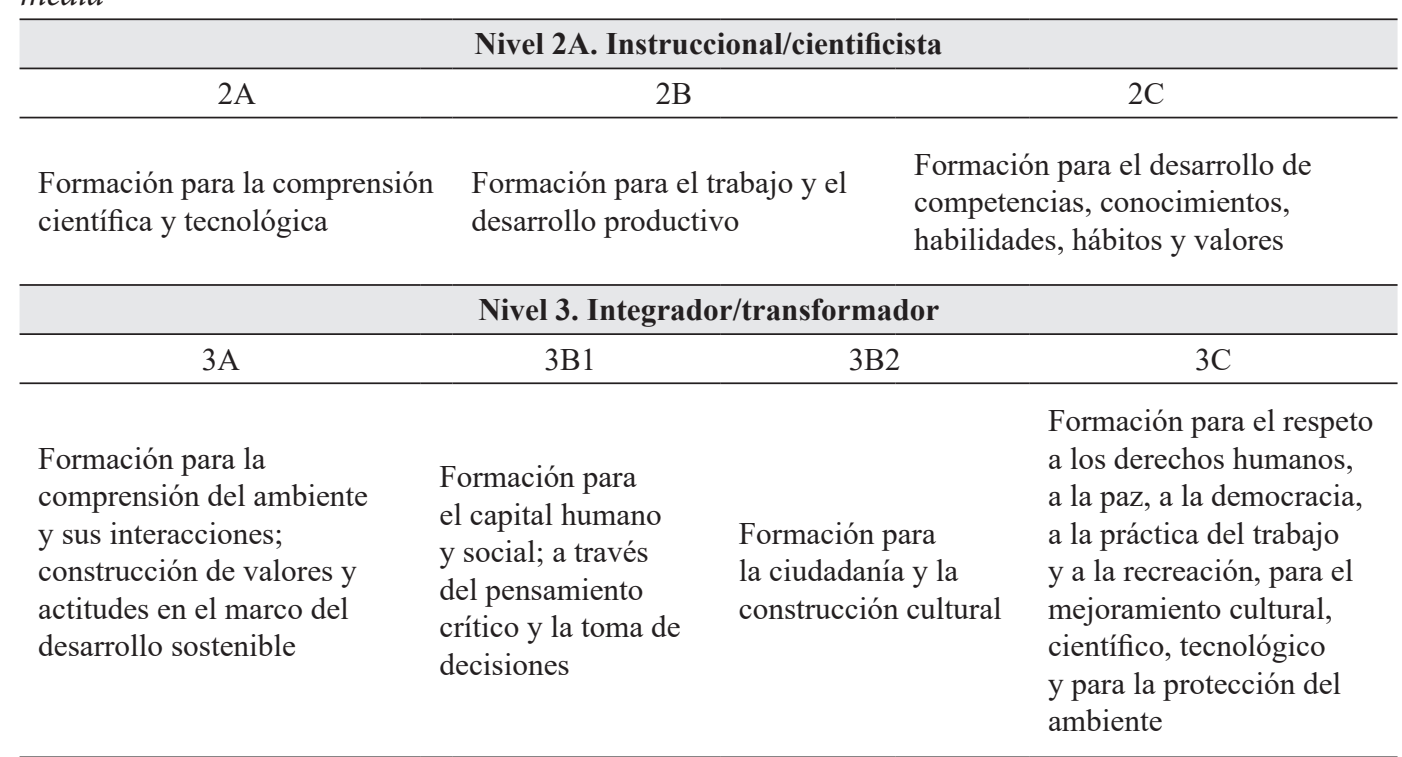


Igualmente, en la tabla 3 se indican las subcategorías referidas a las finalidades del conocimiento escolar en ciencias con su respectiva complejidad.

Tabla 3

Niveles de complejidad. Subcategorías referidas a finalidades del conocimiento escolar en ciencias

\begin{tabular}{|c|c|c|c|}
\hline \multicolumn{4}{|c|}{ Nivel 1. Tradicional } \\
\hline \multicolumn{4}{|c|}{1} \\
\hline \multicolumn{4}{|c|}{$\begin{array}{l}\text { Formación científica entendida como incorporación de términos especializados —a modo de etiquetas-, } \\
\text { proporcionar las informaciones fundamentales de la cultura vigente }\end{array}$} \\
\hline \multicolumn{4}{|c|}{ Nivel 2A. Instruccional/cientificista } \\
\hline \multicolumn{4}{|c|}{$2 \mathrm{~A}$} \\
\hline \multicolumn{4}{|c|}{$\begin{array}{l}\text { Formación para proporcionar un aprendizaje "moderno" y "eficaz", siguiendo una secuencia } \\
\text { predeterminada por los expertos en la pretensión de ser científicos — pequeños científicos-. }\end{array}$} \\
\hline \multicolumn{4}{|c|}{ Nivel 3. Integrador/ transformador } \\
\hline $3 \mathrm{~A}$ & 3B1 & $3 \mathrm{~B} 2$ & $3 \mathrm{C}$ \\
\hline $\begin{array}{l}\text { Formación } \\
\text { para } \\
\text { comprender } \\
\text { los } \\
\text { fenómenos } \\
\text { que ocurren } \\
\text { en el mundo. }\end{array}$ & $\begin{array}{l}\text { Formación para } \\
\text { el desarrollo de } \\
\text { pensamiento } \\
\text { científico; } \\
\text { desarrollo de } \\
\text { pensamiento } \\
\text { ético y desarrollo } \\
\text { de pensamiento } \\
\text { estético. }\end{array}$ & $\begin{array}{l}\text { Formación para } \\
\text { entender el papel } \\
\text { de la ciencia y } \\
\text { la tecnología. } \\
\text { Desarrollar } \\
\text { actitudes de } \\
\text { responsabilidad } \\
\text { con el ambiente. }\end{array}$ & $\begin{array}{l}\text { Formación para el enriquecimiento progresivo } \\
\text { del conocimiento del estudiante y su proceso } \\
\text { formativo hacia modelos más complejos } \\
\text { de entender el mundo y de actuar en él. } \\
\text { Formación de sujetos críticos, propositivos } \\
\text { y argumentativos, capaces de producir } \\
\text { explicaciones sobre los fenómenos de la } \\
\text { naturaleza, así como de proponer y aplicar } \\
\text { estrategias para la solución de problemáticas } \\
\text { relevantes del contexto socioculturales y } \\
\text { ambientales. }\end{array}$ \\
\hline
\end{tabular}

Finalmente, para una mejor comprensión de los resultados, puede verse la tabla 4 con los nombres de cada documento: la sigla asignada, el orden dado al documento en la unidad hermenéutica y un ejemplo de identificador numérico para las citas respectivas.

\section{Tabla 4}

Documentos analizados en la unidad hermenéutica (UH)

\begin{tabular}{cccc}
\hline $\begin{array}{c}\text { Orden de la } \\
\text { entrevista en } \\
\text { UH }\end{array}$ & $\begin{array}{c}\text { Nombre del documento de la } \\
\text { entrevista }\end{array}$ & Sigla & $\begin{array}{c}\text { Designación de la entrevista } \\
\text { para presentación de los } \\
\text { resultados }\end{array}$ \\
\hline P1 & $\begin{array}{c}\text { Lineamientos Curriculares- } \\
\text { Ciencias Naturales y Educación } \\
\text { Ambiental }\end{array}$ & LCNEA & D1 \\
\hline P2 & $\begin{array}{c}\text { Cuadro anexo. Lineamientos } \\
\text { Curriculares-Ciencias Naturales y } \\
\text { Educación Ambiental }\end{array}$ & LCNEA & D2 \\
\hline P3 & $\begin{array}{c}\text { Estándares Básicos de } \\
\text { Competencias en Ciencias } \\
\text { Naturales }\end{array}$ & ECN & D3 \\
\hline P4 & $\begin{array}{c}\text { Derechos Básicos del Aprendizaje. } \\
\text { Fundamentación teórica }\end{array}$ & DCN \\
\hline P5 & $\begin{array}{c}\text { Derechos Básicos de Aprendizaje } \\
\text { de grado } 1^{\circ} \text { a grado 11 }\end{array}$ & DCN & D5 \\
\hline P6 & $\begin{array}{c}\text { Derechos Básicos de } \\
\text { Aprendizaje. Documento } \\
\text { para la implementación de los } \\
\text { DBA. Mallas de Aprendizaje. } \\
\text { Presentación general y por áreas. } \\
\text { Ejemplo de Malla de Aprendizaje } \\
\text { (grado 5 }{ }^{\circ} \text { ) }\end{array}$ & DCN & D6 \\
\hline
\end{tabular}




\begin{tabular}{cccc}
\hline P7 & $\begin{array}{c}\text { Entrevista coautora. Lineamientos } \\
\text { curriculares-Ciencias Naturales y } \\
\text { Educación Ambiental. }\end{array}$ & $\begin{array}{c}\text { Entrevista } \\
\text { LCNEA }\end{array}$ & ENT-LCNEA \\
\hline P8 & $\begin{array}{c}\text { Entrevista coautor. Estándares } \\
\text { Básicos de Competencias en } \\
\text { Ciencias Naturales y Sociales. }\end{array}$ & $\begin{array}{c}\text { Entrevista } \\
\text { ECN }\end{array}$ & ENT-ECN \\
& $\begin{array}{c}\text { Entrevista coautora. Derechos } \\
\text { Básicos del Aprendizaje. Ciencias } \\
\text { Paturales. }\end{array}$ & $\begin{array}{c}\text { Entrevista } \\
\text { DCN }\end{array}$ & ENT-DCN \\
\hline
\end{tabular}

\section{Resultados}

El propósito es responder la pregunta: ¿cuáles son yquébuscan las finalidades del conocimiento escolar en ciencias en los LCNEA (D1 y D2), los ECN (D3) y DCN (D4, D5 y D6)?

La tabla 5 muestra la recurrencia de citas para cada una de las subcategorías de la macrocategoría finalidades. Para el caso de las generales de la educación básica y media, se destacan las siguientes cuatro finalidades:

1. Respetar los derechos, incluidos los de las personas - por ejemplo: recreación-, la sociedad — por ejemplo: la paz—y el ambiente — por ejemplo: la protección de los recursos-.

2. Comprender el entorno natural y los tipos de interacciones que se dan para el desarrollo de valores y cambios de actitud de las relaciones ser humano-naturaleza.

3. Formación general para la comprensión del ambiente y sus interacciones y, además, forjar bases para que el sujeto pueda desempeñarse en lo laboral y en el mundo productivo.

4. Desarrollar competencias, habilidades, hábitos y valores.

Cabe recordar que estas finalidades se plantean en los fines de la educación, en los objetivos educativos por niveles y en el papel de la escuela en la formación de la educación básica y media.

Para el caso de las finalidades específicas del conocimiento escolar en ciencias, se destacan cuatro:

1. Las relacionadas con las formas de proceder de la comunidad científica para lograr el desarrollo del pensamiento científico - por ejemplo: formulación de preguntas, hipótesis, diseño de experimentos, entre otros-.

2. La formación de ciudadanos y ciudadanas como eje central, que privilegie el desarrollo crítico y reflexivo. El conocimiento de la ciencia, la tecnología y la sociedad están al servicio de la comprensión de los fenómenos y las problemáticas socioculturales y ambientales. Se busca el desarrollo de valores y actitudes en coherencia con unas prácticas en pro del bienestar comunitario, para desarrollar pensamiento crítico y reflexivo.

3. Las que enfatizan comprender el papel de los desarrollos de la ciencia y la tecnología en la sociedad. Esto implica no solo reconocer la ciencia y la tecnología como productos sociales y culturales, sino además analizar los beneficios y los perjuicios para poder tomar decisiones informadas al respecto, tanto de forma individual como colectiva. 
4. Las que ponen en diálogo el desarrollo del pensamiento científico, sin desligarlo del desarrollo del pensamiento ético y estético: cómo desde la comprensión de los fenómenos se generan valores de respeto y la posibilidad de apreciar el valor de los elementos del entorno para el bienestar.

Tabla 5

Recurrencia de citas de las subcategorías de la categoría finalidades educativas

\begin{tabular}{|c|c|c|c|c|}
\hline \multirow[b]{2}{*}{ Subcategorías de la macrocategoría finalidades educativas } & \multicolumn{3}{|c|}{ Documentos } & \multirow[b]{2}{*}{ Total } \\
\hline & $\begin{array}{l}\text { D1,2, } \\
\text { P7 }\end{array}$ & $\begin{array}{l}\text { D3, } \\
\text { P8 }\end{array}$ & $\begin{array}{l}\text { D4,5,6, } \\
\text { P9 }\end{array}$ & \\
\hline \multicolumn{5}{|l|}{ Generales para la educación básica y media } \\
\hline Formación para la ciudadanía y la construcción cultural. & 2 & 2 & 0 & 4 \\
\hline Formación para la comprensión científica y tecnológica. & 5 & 0 & 0 & 5 \\
\hline $\begin{array}{l}\text { Formación para el desarrollo de competencias, conocimientos, } \\
\text { habilidades, hábitos y valores. }\end{array}$ & 4 & 2 & 3 & 9 \\
\hline $\begin{array}{l}\text { Formación general para la comprensión del ambiente y sus } \\
\text { interacciones; construcción de valores y actitudes en el marco del } \\
\text { desarrollo sostenible. }\end{array}$ & 5 & 1 & 0 & 6 \\
\hline $\begin{array}{l}\text { Formación para el capital humano y social; a través del } \\
\text { pensamiento crítico y la toma de decisiones. }\end{array}$ & 2 & 0 & 1 & 3 \\
\hline $\begin{array}{l}\text { Formación para el respeto a los derechos humanos, a la paz, } \\
\text { a la democracia, a la práctica del trabajo y a la recreación, } \\
\text { para el mejoramiento cultural, científico, tecnológico y para la } \\
\text { protección del ambiente. }\end{array}$ & 10 & 1 & 0 & 11 \\
\hline Formación para el trabajo y el desarrollo productivo. & 6 & 0 & 0 & 6 \\
\hline
\end{tabular}

Para el conocimiento escolar en ciencias naturales — particulares del área-

\begin{tabular}{|c|c|c|c|c|}
\hline $\begin{array}{l}\text { Formación científica entendida como incorporación de términos } \\
\text { especializados - a modo de etiquetas- }- \text {. Proporcionar las } \\
\text { informaciones fundamentales de la cultura vigente. }\end{array}$ & 1 & 0 & 0 & 1 \\
\hline $\begin{array}{l}\text { Formación para proporcionar un aprendizaje "moderno" } \\
\text { y "eficaz", siguiendo una secuencia predeterminada por } \\
\text { los expertos en la pretensión de ser científicos — pequeños } \\
\text { científicos-. }\end{array}$ & 45 & 5 & 10 & 60 \\
\hline $\begin{array}{l}\text { Formación para educar al estudiante al involucrarlo en la } \\
\text { realidad inmediata, en dos sentidos: satisfacer los deseos de } \\
\text { los estudiantes para que se sientan bien; o tener en cuenta sus } \\
\text { intereses para el desarrollo de la autonomía y la creatividad. }\end{array}$ & 1 & 0 & 0 & 1 \\
\hline
\end{tabular}

Formación para el enriquecimiento progresivo del conocimiento del estudiante hacia modelos más complejos de entender el mundo y de actuar en él. Formación de sujetos críticos, propositivos y argumentativos, capaces de producir explicaciones sobre los fenómenos de la naturaleza, así como de proponer y aplicar estrategias para la solución de problemáticas.

\begin{tabular}{lllll}
\hline $\begin{array}{l}\text { Formación para comprender los fenómenos que ocurren en el } \\
\text { mundo. }\end{array}$ & 3 & 2 & 0 & $\mathbf{5}$ \\
\hline $\begin{array}{l}\text { Formación para entender el papel de la ciencia y la tecnología; } \\
\text { desarrollar actitudes de responsabilidad con el ambiente. }\end{array}$ & 8 & 4 & 5 & $\mathbf{1 7}$ \\
\hline $\begin{array}{l}\text { Formación para el "desarrollo de pensamiento científico"; } \\
\text { desarrollo de pensamiento ético y desarrollo de pensamiento } \\
\text { estético. }\end{array}$ & 7 & 5 & 2 & $\mathbf{1 4}$ \\
\hline
\end{tabular}


Nota. D1: Lineamientos Curriculares. Área de Ciencias Naturales y Educación Ambiental (LCNEA); D2: Cuadro anexo de los LCNEA. Ejemplos de Estructuración Curricular en el Área de Ciencias Naturales y Educación Ambiental; D3: Estándares Básicos de Competencias en Ciencias Naturales (ECN); D4: Documento. Fundamentación teórica de los DBA (DCN); D5: Derechos Básicos del Aprendizaje. Ciencias Naturales (DCN); D6: Documento para la implementación de los DBA. Mallas de Aprendizaje. Presentación general y por áreas. Ejemplo de Malla de Aprendizaje (grado $5^{\circ}$ ).

Como se aprecia en los LCNEA, las finalidades del conocimiento escolar en ciencias buscan principalmente el desarrollo de pensamiento científico, el aporte a la formación de ciudadanos, la comprensión del papel de la ciencia y la tecnología en el desarrollo de las sociedades y la vinculación del desarrollo de pensamiento científico con el pensamiento ético y estético. Lo anterior aportaría a finalidades generales de la educación que enfatizan en el respeto a los derechos personales, sociales y del ambiente; a lograr mejorar las relaciones ser humano-naturaleza; al desarrollo de habilidades, hábitos y valores; y además a preparar a los estudiantes para desempeñarse en el ámbito laboral y productivo.

Que el estudiante desarrolle un pensamiento científico que le permita contar con una teoría integral del mundo natural dentro del contexto de un proceso de desarrollo humano integral, equitativo y sostenible que le proporcione una concepción de sí mismo y de sus relaciones con la sociedad y la naturaleza armónica con la preservación de la vida en el planeta. (MEN, 1998, p. 66)

Por su parte, los ECN enfatizan en la formación de ciudadanos, en el desarrollo de un pensamiento científico, y en la construcción del conocimiento al integrar tres ejes: "me aproximo al conocimiento como científico natural"; "manejo conocimientos propios de las ciencias naturales" y "desarrollo compromisos personales y sociales", (MEN, 2006, p. 114) en pro de la comprensión de situaciones, de la aplicación de conocimientos y de la consolidación de valores personales y sociales. Estas se relacionan con las metas generales que pretenden el desarrollo de competencias, habilidades y valores para la vida, la convivencia, la productividad y para seguir aprendiendo. Se destaca lo referente a una ciudadanía respetuosa y comprometida con el bien común.

Ha de ser meta de la formación en ciencias - tanto sociales como naturales- desarrollar el pensamiento científico y en consecuencia fomentar la capacidad de pensar analítica y críticamente. Solamente así, podremos contar con una generación que estará en capacidad de evaluar la calidad de la información a la que accede - en términos de sus fuentes y la metodología utilizada - ... que contará con los elementos para identificar y buscar solución a los problemas y que estará atenta a proceder de manera rigurosa. Se trata, entonces, de "desmitificar" las ciencias y llevarlas al lugar donde tienen su verdadero significado, llevarlas a la vida diaria, a explicar el mundo en el que vivimos. (MEN, 2006, pp. 27-28)

Para el caso de los DCN, las finalidades específicas se centran en la comprensión de la naturaleza de las ciencias, en el desarrollo de habilidades y actitudes científicas y en la interacción de las ciencias con otras disciplinas como las matemáticas y la ingeniería para la construcción de conocimiento científico y, a la vez, para el desarrollo de habilidades, actitudes y normas relacionadas con lo ético, lo estético y lo cultural (Universidad de Antioquia \& MEN, 2016). Estas aportarán a finalidades generales relacionadas con la formación integral, en donde se pone en juego un saber, un saber hacer y un saber ser, 
que serán las bases para otros aprendizajes y la movilización de pensamientos, acciones, actitudes y valores personales y colectivos.

Entonces el rango que se busca en los DBA es aportar no solo a la comprensión de conceptos, fenómenos, hechos científicos, sino al desarrollo de habilidades de pensamiento referidas especialmente a la investigación, la representación y la comunicación; así mismo, a la promoción de actitudes científicas y ambientales, que apunten a la comprensión de la naturaleza de la ciencia, los procesos que siguen los científicos, y al desarrollo de valores, actitudes y acciones en correspondencia con premisas de convivencia, democracia, solidaridad y sostenibilidad ambiental. (Universidad de Antioquia \& MEN, 2016, p. 23)

Entre los elementos que comparten las finalidades específicas entre los LCNEA, los ECN y los DCN, está el desarrollo de pensamiento científico, que tiene como referente central el conocimiento científico y las formas de proceder de la comunidad científica. Sin embargo, hay un avance en proponer una formación en ciencias que permita asumir una postura diferente como individuos y como ciudadanos ante situaciones de la vida cotidiana y social - con mayor énfasis en los ECN-. También se destaca el aporte del conocimiento científico en la comprensión de situaciones y fenómenos del contexto - con mayor relevancia los LCNEA a través de la propuesta del mundo de la vida como punto de partida y de llegada-. Igualmente se plantea que la formación en ciencias deberá posibilitar la complejidad del conocimiento cotidiano - con mayor explicación en los DCN desde uno de los propósitos de la ciencia escolar-.

Se puede apreciar que las finalidades para el conocimiento escolar en ciencias en las orientaciones trascienden de visiones centradas en la ciencia, sus conceptos y formas de actuación, hacia otras de carácter más contextual en el que la relación con la sociedad (Martínez \& Molina, 2011; Oliveira \& Salgado, 2020; Roberts \& Bybee, 2014) permite identificar al conocimiento escolar como un conocimiento diferenciado epistemológicamente (García, 1998; García-Pérez, 2015).

Así, estas propuestas abordan finalidades que parecen no solo centrarse en el conocimiento científico; en cambio, parecen sensibles a otros conocimientos (Martínez \& Molina, 2011). De esta manera, se reconoce la especificidad del contexto escolar y de los procesos creativos que permiten producirlo o de procesos de recontextualización (Wallace \& Priestley, 2017).

\section{Conclusión}

En esta caracterización de las finalidades, se encontró que los LCNEA, los ECN y los DCN se enmarcan en las finalidades generales que se ubican principalmente en la Ley 115 (1994). Esta característica es uno de los planteamientos propuestos por Lemke (2006) cuando reflexiona acerca del aporte que debe hacer la educación científica en el siglo XXI:

Las metas de la educación científica deben ser formuladas dentro del contexto de nuestras metas más amplias para la educación en general y de nuestra definición de aquello que contribuye a una mejor sociedad y a una vida mejor para todas las personas. (p. 6)

Como se aprecia en la presentación de los resultados de la caracterización de las finalidades, el para qué del conocimiento escolar en ciencias en nuestro país se centra en aportar a la construcción de una ciudadanía responsable, con un énfasis mayor en los ECN 
y en los LCNEA. Lo anterior concuerda con lo propuesto por Acevedo (2004) y Hodson (2003, 2013), quienes plantean que la educación científica deberá aportar en y para la ciudadanía (Roberts \& Bybee, 2014) desde el desarrollo de una actitud crítica (González et al., 2021), a diferencia de los resultados analizados en Chile del programa Enseñanza de las Ciencias Basada en la Indagación, en los que se identifica la prevalencia de objetivos cientificistas (Castillo \& Lopes, 2020).

Es de destacar que el énfasis del aporte del conocimiento escolar en ciencias para lograr una ciudadanía responsable, en los ECN, se relaciona con la contribución de las ciencias sociales que, para el caso de este documento, comparten un marco conceptual de base. Esto se ratifica desde la tesis doctoral de Calderón (2007), que da cuenta de las perspectivas epistemológicas del conocimiento escolar en los lineamientos curriculares y en los textos escolares de ciencias sociales en Colombia. Como resultados, el autor dice: "la intencionalidad de formación más reiterada en los documentos oficiales es la formación ciudadana, ella se constituye en propósito clave de la formación en ciencias sociales” (p. 460). Así, de acuerdo con los documentos analizados, se señala que, en el caso de las finalidades, estas se alejan de visiones académicas que buscan garantizar la transmisión de los conocimientos que la humanidad ha producido (Lopes \& Macedo, 2011) o de una visión tradicional instrumental cientificista, centrada en la adquisición de conceptos y procedimientos propios de la ciencia (Martínez, 2000; Martínez \& Valbuena, 2013).

Otro aspecto evidente en las finalidades del conocimiento escolar en ciencias, según las orientaciones, es el desarrollo del pensamiento científico en procesos de construcción de las ciencias y de las personas que hacen ciencia. Esto también se evidenció en el estudio de Criado et al. (2014), quienes analizan el currículo español oficial de ciencias con el de Gales y Estados Unidos. Uno de los aspectos allí abordados son las finalidades educativas a través de los siguientes indicadores:

1.1 La adquisición de conocimientos científico-tecnológicos básicos. 1.2 El desarrollo de habilidades y destrezas próximas a la actividad científica. 1.3 Una primera comprensión de elementos básicos de la Naturaleza de la Ciencia (NDC). 1.4 El desarrollo de un pensamiento crítico y responsable ante cuestiones sociocientíficas significativas. (p. 252)

Para el caso de los documentos en Colombia, este aspecto es mucho más explícito según la actualidad del documento. En los DCN se retoma lo propuesto por Harlen et al. (2015) frente a los propósitos y metas de la educación en ciencias: "la comprensión de la naturaleza de las ciencias" (p. 23). Igualmente, se detalla el tipo de habilidades científicas, organizadas en tres ejes: investigación, representación y comunicación. Es importante aclarar que el desarrollo del pensamiento científico en ninguno de los documentos busca una formación centrada únicamente en la ciencia, sino que esta formación estará al servicio de comprender y asumir una postura frente a los desarrollos de la ciencia y la tecnología; de tener una visión mucho más compleja de las interacciones en el entorno; de consolidar valores y actitudes frente a las relaciones ser humano-naturaleza; y por supuesto, de ser la base para continuar con carreras que estén relacionadas con las ciencias o para enfrentarse al ámbito laboral y productivo.

Sin embargo, cabe preguntarse:

- ¿cómo se relaciona esta finalidad de formación de ciudadanos con los contenidos 
propuestos?

- ¿qué papel se concede a la relación entre los diferentes conocimientos - científico, tecnológico, cotidiano, cultural, etc.- en el conocimiento escolar propuesto en estas orientaciones?

- ¿cuáles son los criterios para legitimar el conocimiento escolar en ciencias?

- ¿qué papel cumplen las normativas como la Ley General de Educación o las problemáticas socioculturales y ambientales?

- ¿de qué manera es posible evidenciar los procesos de complejidad que se enuncian en las finalidades en relación con los contenidos propuestos?

Estas preguntas son motivo para hacer un análisis más amplio en relación con otras categorías como los contenidos escolares, los tipos y niveles de organización de los contenidos escolares, los referentes epistemológicos del conocimiento escolar y los criterios de validación del conocimiento escolar.

\section{Referencias}

Acevedo, J. (2004). Reflexiones sobre las finalidades de la enseñanza de las ciencias: educación científica para la ciudadanía. Revista Eureka sobre Enseñanza y Divulgación de las Ciencias, 1(1), 3-16.

Aikenhead, G. (1985). Science curricula and social responsibility. En R. Bybee (Ed.), ScienceTechnology-Society (pp. 128-143). NSTA.

Álvarez, A. (2007). Las Ciencias Sociales en el currículo escolar: Colombia 1930-1960 [Tesis doctoral, Universidad Nacional de Educación a Distancia]. Repositorio de contenidos digitales. http://62.204.194.43/fez/view/tesisuned:Educacion-Aalvarez

Álvarez, A. (2015). Del saber pedagógico a los saberes escolares. Pedagogía y Saberes, 42, 21-29. https://doi.org/10.17227/01212494.42pys21.29

Banet, E. (2007). Finalidades de la educación científica en secundaria: opinión del profesorado sobre la situación actual. Enseñanza de las Ciencias, 25(1), 5-20.

Bardín, L. (1996). Análisis del contenido. Akal.

Bybee, R., \& Deboer, G. (1994). Research on Goals for the Science Curriculum. En D. Gabel (Ed.), Handbook of Research on Science Teaching and Learning (pp. 357-387). Macmillan.

Calderón, J. (2007). Perspectivas epistemológicas y didácticas del conocimiento escolar en los Lineamientos Curriculares y en los textos escolares de Ciencias Sociales de la educación en Colombia: análisis de casos [Tesis Doctoral, Universidad de Sevilla]. Depósito de Investigación Universidad de Sevilla.

Cárdenas, A., \& Martínez, C. (2017). Los referentes curriculares instituidos para la elaboración del conocimiento escolar en ciencias en Colombia: ¿qué caracteriza la estructura de los estándares básicos de competencias en Ciencias? Enseñanza de las Ciencias, (extraordinario), 1183-1187.

Cárdenas, A., \& Martínez, C. (2018). Las finalidades de la enseñanza de las ciencias 
naturales desde el punto de vista de profesores en ejercicio en Bogotá. Una aproximación al problema. Tecné, Episteme y Didaxis: TED, (extraordinario), 1-7. https://revistas. pedagogica.edu.co/index.php/TED/article/view/9026

Castillo, S. \& Lopes, A. (2020). Traducción en el discurso cientificista: el caso del programa ECBI (Enseñanza de la Ciencia Basada en la Indagación) en Chile. En A. Molina (Comp.), Investigación y formación de profesores de ciencias: diálogos de perspectivas latinoamericanas (pp. 131-159). Universidad Distrital Francisco José de Caldas.

Chamizo, J., \& Pérez, Y. (2017). Sobre la enseñanza de las ciencias naturales. Revista Iberoamericana de Educación, 74(1), 23-40. https://doi.org/10.35362/rie741624

Chernobilsky, L. (2006). El uso de la computadora como auxiliar en el análisis de datos cualitativos. En I. Vasilachis (Coord.), Estrategias de investigación cualitativa (pp. 239271). Gedisa.

Congreso de Colombia. (1994, 8 de febrero). Ley 115. Ley General de Educación de 1994. https://www.mineducacion.gov.co/1621/articles-85906_archivo_pdf.pdf

Congreso de Colombia. (1995, 5 de junio). Ley 188. Plan Nacional de Desarrollo e Inversiones 1995-1998. Diario Oficial n ${ }^{\circ} .41 .876$ http://www.secretariasenado.gov.co/senado/ basedoc/ley_0188_1995.html

Congreso de Colombia. (2001, 21 de diciembre). Ley 715. Por la cual se dictan normas orgánicas en materia de recursos y competencias de conformidad con los artículos 151, 288, 356 y 357 (Acto Legislativo 01 de 2001) de la Constitución Política y se dictan otras disposiciones para organizar la prestación de los servicios de educación y salud, entre otros. https://www.mineducacion.gov.co/1621/articles-86098_archivo_pdf.pdf

Criado, A., Cruz, M., García, A., \& Cañal, P. (2014). ¿Cómo mejorar la educación científica de primaria en España desde el currículo oficial? Sugerencias a partir de un análisis curricular comparativo en torno a las finalidades y contenidos de la ciencia escolar. Enseñanza de las Ciencias, 32(3), 249-266

Departamento Nacional de Planeación. (2015). Plan Nacional de Desarrollo 2014-2018. https://colaboracion.dnp.gov.co/cdt/pnd/pnd\%202014-2018\%20tomo\%201\%20 internet.pdf

Furió, C., Vilches, A., Guisasola, J., \& Romo, V. (2001). Finalidades de la enseñanza de las ciencias en la Secundaria Obligatoria. ¿Alfabetización científica o preparación propedéutica? Enseñanza de las Ciencias, 19(3), 365-376.

García, E. (1998). Hacia una teoría alternativa sobre los contenidos escolares. Díada.

García-Pérez, F. (2015). El conocimiento escolar en el centro del debate didáctico. Reflexiones desde la perspectiva docente. Con-Ciencia Social, 19, 49-62.

Gil, D., \& Vilches, A. (2001). Una alfabetización científica para el siglo XXI. Obstáculos y propuestas de actuación. Investigación en la Escuela, 43, 27-37.

Gil, D., \& Vilches, A. (2004). Contribución de la ciencia a la cultura ciudadana. Cultura y Educación, 16(3), 259-272 
González, F., Blanco, Á., \& España, E. (2021). The Nature of Science and Citizenship: a Delphi Analysis. Research in Science Education, 51, 791-818. https://doi.org/10.1007/ s11165-018-9817-5

Goodson, I. (1991). La construcción social del currículum, posibilidades y ámbitos de investigación de la historia del currículum. Revista de Educación, 295, 7-37. https:// dialnet.unirioja.es/servlet/articulo?codigo $=18853$

Harlen, W., Bell, D., Devés, R., Dyasi, H., Fernández, G., Léna, R., Reiss, M., Rowell, P., \& Yu, W. (2015). Trabajando con las grandes ideas de la educación en ciencias. Programa de Educación en Ciencias de la IAP.

Hodson, D. (1993). Re-Thinking Old Ways: Towards a More Critical Approach to Practical Work in School Science. Studies in Science Education, 22, 85-142.

Hodson, D. (2003). Time for action: science education for an alternative future. International Journal of Science Education, 25(6), 645-670.

Hodson, D. (2013). La educación en ciencias como un llamado a la acción. Archivos de Ciencias de la Educación, 7(7), 1-15.

Kyle, W. (1996). Editorial: The importance of investing in human resources. Journal of Research in Science Teaching, 33, 1-4.

Lemke, J. (2006). Investigar para el futuro de la educación científica: nuevas formas de aprender, nuevas formas de vivir. Enseñanza de las Ciencias, 24(1), 5-12

Lopes, A. (2005). Discursos curriculares na disciplina escolar química. Ciência \& Educação, 11(2), 263-278.

Lopes, A. (2008). Articulaciones en las políticas de currículo. Perfiles Educativos, 30(120), 63-78.

Lopes, A., \& Macedo, E. (2011). Teorías de currículo. Apoio Faperj.

Martínez, C. (2000). Las propuestas curriculares sobre el conocimiento escolar en el área de conocimiento del medio: dos estudios de caso en profesores de primaria [Tesis Doctoral, Universidad de Sevilla]. Depósito de Investigación Universidad de Sevilla.

Martínez, C. (2005). Las propuestas de conocimiento escolar en los inicios del aprendizaje de la química: un estudio de caso en las clases de ciencias en sexto grado de educación primaria. Universidad del Tolima

Martínez, C. (2016). El conocimiento profesional de profesoras de ciencias de primaria sobre el conocimiento escolar: dos estudios de caso, en Aulas Vivas y Aulas Hospitalarias del Distrito Capital de Bogotá. Universidad Distrital Francisco José de Caldas.

Martínez, C. (2017). Ser maestro de ciencias: productor de conocimiento profesional y de conocimiento escolar. Universidad Distrital Francisco José de Caldas.

Martínez, C., \& Molina, A. (2011). La especificidad del conocimiento profesional y del conocimiento escolar en las clases de ciencias: algunas relaciones con la cultura. Revista EDUCyT, 3, 36-58. https://acortar.link/2jTRaJ

Martínez, C., \& Valbuena, E. (2013). El conocimiento profesional de los profesores de ciencias 
sobre el conocimiento escolar. Universidad Distrital Francisco José de Caldas.

Martínez, C., Cárdenas, A., \& Jirón, M. (2020). Currículos oficiales en ciencias y conocimiento escolar: una revisión desde publicaciones especializadas. En T. Sánchez, T. Durango, \& M. Casallas (Comps.), Encuentro de socialización de experiencias investigativas en la Facultad de Ciencias y Educación Memorias. Número especial. Evaluación, currículo y formación docente (pp. 193-204). UD Editorial.

Martínez, C., Lopes, A., Cárdenas, A., \& Jirón, M. (2016). Las propuestas de conocimiento escolar en ciencias naturales en las orientaciones curriculares de la Secretaría de Educación de Bogotá (2007-2015) [Proyecto de investigación]. Centro de Investigaciones y Desarrollo Científico; Universidad Distrital Francisco José de Caldas.

Mason, J. (1996). Qualitative Researching. Sage.

MEN. (1998). Lineamientos curriculares. Ciencias Naturales y Educación Ambiental. Delfín Ltda.

MEN. (2003). La revolución educativa. Plan sectorial 2002-2006. https://www.mineducacion. gov.co/1621/articles-85266_archivo_pdf.pdf

MEN. (2006). Estándares Básicos de Competencias en Lenguaje, Matemáticas, Ciencias y Ciudadanas. Imprenta Nacional de Colombia.

MEN. (2015, 25 de febrero). Decreto 0325. Por el cual se establece el Día de la Excelencia Educativa en los establecimientos educativos de preescolar, básica y media, y se dictan otras disposiciones. http://www.seduca.gov.co/component/phocadownload/category/31decretos?download=3034:decreto-0325-de-2015-dia-de-la-excelencia-educativa

MEN. (2016). Derechos Básicos de Aprendizaje. Panamericana.

Merchán, F. (2002). El estudio de la clase de historia como campo de producción del currículo. Enseñanza de las Ciencias Sociales, 1, 41-54. https://dialnet.unirioja.es/servlet/ articulo? codigo $=500419$

Merchán, F., \& García, F. (1994). El Proyecto IRES: una alternativa para la transformación escolar. Signos de una Nueva Generación, 13(5), 58-69.

Moraes, R., \& Galiazzi, M. (2006). Análise textual discursiva: processo reconstrutivo de múltiplas faces. Ciência \& Educação, 12(1), 117-12.

Ottander, K., \& Simon, S. (2021). Learning democratic participation? Meaning-making in discussion of socioscientific issues in science education, International Journal of Science Education, 43(12), 1895-1925. https://doi.org/10.1080/09500693.2021.1946200

Oliveira, R., \& Salgado, S. (2020). A Educação em Direitos Humanos no Ensino de Ciências em interface com a teoria do Giro Decolonial: uma análise. Ensino em Re-vista, 27(2), 698-726. http://dx.doi.org/10.14393/ER-v27n2a2020-14

Paixao, M., \& Cachapuz, A. (1999). La enseñanza de las ciencias y la formación de profesores de enseñanza primaria para la reforma curricular: de la teoría a la práctica. Enseñanza de las Ciencias, 17(1), 69-77.

Porlán, R. (2018). Didáctica de las ciencias con conciencia. Enseñanza de las Ciencias, 36(3), 


\section{5-22. https://doi.org/10.5565/rev/ensciencias.2795}

Roa, P. (2015). La emergencia de la biología en la escuela colombiana: una mirada genealógica entre 1900-1930. Pedagogía y Saberes, 42, 145-151. https://doi.org/10.17227 /01212494.42pys145.151

Roberts, D., \& Bybee, R. (2014). Scientific literacy, science literacy, and science education. En N. Lederman, \& S. Abell (Eds.), Handbook of Research on Science Education. Vol. II (pp. 545-558). Routledge

Stake, R. (1999). Estudio de casos. En N. Denzin, \& Y. Lincoln (Eds.), Handbook of Qualitative Research (pp. 236-247). Sage Publications.

Universidad de Antioquia, \& MEN. (2016). Fundamentación teórica de los DBA Producto Nro. 11: Documento con la fundamentación teórica de los DBA que indique justificación, antecedentes, referentes legales, teóricos, conceptuales que sustentan la propuesta. [Contrato Interadministrativo 0803 de 2016].

Vasilachis, I. (2006). La investigación cualitativa. En I. Vasilachis (Coord.), Estrategias de investigación cualitativa (pp. 23-60). Gedisa.

Vázquez, A., Acevedo, J., \& Massanero, M. (2005). Más allá de la enseñanza de las ciencias para científicos: hacia una educación científica humanística. Revista Electrónica de Enseñanza de las Ciencias, 4(2), 1-30.

Wallace, C., \& Priestley, M. (2017). Secondary Science Teachers as Curriculum Makers: Mapping and Designing Scotland's New Curriculum for Excellence. Journal of Research in Science Teaching, 54(3), 324-349. https://doi.org/10.1002/tea.21346 